\title{
Disparities in Secondary Prevention of Atherosclerotic Heart Disease After Coronary Artery Bypass Grafting in Northern Plains American Indians
}

Hannah Kruger, ${ }^{1, \dagger}$ Christopher Zumwalt, ${ }^{1, \dagger}$ Rory Guenther, Rick Jansen, ${ }^{2}$ Donald Warne, and Cornelius Dyke ${ }^{4, *}$

\begin{abstract}
Introduction: Cardiovascular disease has become the leading cause of death in American Indians (Als). For patients with severe disease requiring coronary artery bypass grafting (CABG), Als have been demonstrated to present with increased risk factors. Guideline-directed medical therapy after CABG effectively reduces mortality and recurrent ischemic events in all patients and is especially important in high-risk populations such as Als.

Methods: Isolated CABG patients between 2012 and 2017 were studied and 74 Al patients were identified. Propensity matching was performed and the resulting 148 patients were followed for a year after surgery. Guidelinedirected medical therapy (GDMT) for secondary prevention of atherosclerotic disease after CABG was detailed in all patients.

Results: GDMT was similar between groups (85\% Al vs. $89 \%$ non-Al; $p=N S$ ), and the incidence of prescribed antiplatelet medications, beta-blockers, and statins was similar. Als were more likely to receive insulin therapy ( $p=0.002)$ and opioids ( $p=0.03$ ) at discharge, while non-Als were more likely to receive anti-arrhythmic medications ( $p=0.002$ ). One year after discharge, GDMT trended lower in Als (75\% Al vs. $85 \%$ non-Al; $p=0.2)$ and Als were less likely to be on a statin 1 year after surgery ( $81 \%$ Al vs. $93 \%$ non-Al; $p=0.04)$. Opioid use trended higher after 1 year in Als (28\% Al vs. $18 \%$ non-Al; $p=$ NS) and fewer Al patients participated in cardiac rehabilitation (CR) after CABG.

Conclusions: Disparities in GDMT for secondary prevention of coronary artery disease after CABG exist, with fewer Al patients receiving statins and undergoing CR 1 year after surgery. Increased use of opioids in Als is troubling and deserves further investigation. Improved adherence to GDMT would be expected to improve longterm outcomes after CABG in this high risk population.
\end{abstract}

Keywords: cardiovascular disease; prevention; American Indian

\section{Introduction}

American Indians (AIs) have more cardiovascular risk factors than the general population and are at increased risk for developing cardiovascular disease (CVD). ${ }^{1-5}$ CVD has also become the leading cause of death in AIs. ${ }^{1}$ While the cause of this increase in CVD is multi- factorial, disparities in socioeconomic status and societal factors contribute to this increase. ${ }^{5,6}$ Recent data have demonstrated that despite increased cardiovascular risk factors, Northern Plains AIs may undergo coronary artery bypass grafting (CABG) with acceptable and similar short-term outcomes as the general

\footnotetext{
${ }^{1}$ University of North Dakota School of Medicine and Health Sciences—Southeast Campus, Fargo, North Dakota.

${ }^{2}$ University of North Dakota School of Medicine and Health Sciences, Fargo, North Dakota.

${ }^{3}$ Department of Public Health, North Dakota State University, Fargo, North Dakota.

${ }^{4}$ Department of Surgery, University of North Dakota School of Medicine and Health Sciences, Fargo, North Dakota.

'Both authors contributed equally to this manuscript and are co-first authors.

*Address correspondence to: Cornelius Dyke, MD, Department of Surgery, University of North Dakota School of Medicine and Health Sciences, Sanford Health Fargo, 1919 North Elm Street, Fargo, ND 58102, E-mail: cornelius.dyke@und.edu

(C) Hannah Kruger et al. 2019; Published by Mary Ann Liebert, Inc. This Open Access article is distributed under the terms of the Creative Commons License (http://creativecommons.org/licenses/by/4.0), which permits unrestricted use, distribution, and reproduction in any medium, provided the original work is properly cited.
} 
population. ${ }^{7}$ However, patients after CABG remain at risk for progression of native vessel and graft atherosclerosis and are at risk for future adverse ischemic events. Prevention of the progression of atherosclerotic heart disease after surgery is recognized as life-saving and expert consensus guidelines for the secondary prevention of coronary artery disease (CAD) exist ${ }^{8}$ and includes both medical and nonmedical therapies. These therapies improve survival after CABG and are recommended for all patients after coronary revascularization. ${ }^{9,10}$ These recommendations are especially important for vulnerable populations with increased risk factors such as AIs. Whether AI patients receive guideline-directed medical therapy (GDMT) after CABG, however, is unknown.

\section{Methods}

Institutional Review Board approval was obtained from Sanford Health and the University of North Dakota Institutional Review Boards, and a waiver of individual consent was given due to the retrospective, chartreview nature of the study. Indian Health Service (IHS) Institutional Review Board approval was not sought as no patient contact occurred and no IHS records were utilized. Data for this single institutional study were collected retrospectively. All isolated CABG patients from June 1, 2012 to June 1, 2017 were included and 74 AI subjects were identified and propensitymatched with 74 non-AI patients, forming the two study cohorts. AIs were identified via IHSs referral or patient self-identification and patients resided both on and off federally recognized reservation land. All patients received surgery at Sanford Health Fargo and follow-up care was done at various Sanford Health hospitals and offices throughout the Upper Midwest. Patient records, medications, and other therapies were identified retrospectively in the Sanford Health electronic medical record (Epic Systems Corporation; www.EPIC.com). IHS records were not queried or utilized. Adherence to GDMT medications and other therapies were assessed at time of discharge, 4 to 6 weeks after surgery, and 1 year postoperatively. Medications at discharge reflect perioperative care and are less relevant for secondary prevention. Data at 30 days and 1 year were considered to reflect secondary prevention strategies and are reported. Adherence to GDMT was defined as taking or being prescribed antiplatelet medications, a statin, and a beta-blocker at 30 days and 1 year postoperatively. Six AI and three non-AIs were lost to follow-up at 1 year. Propensity matching between the $\mathrm{AI}$ and non-AI groups was done. Due to the limited sample size, the Society of Thoracic Surgeons (STS) composite risk score was used for matching. The STS composite risk score is a wellknown and validated tool which incorporates multiple variables to calculate an overall individual patient risk score (http://riskcalc.sts.org/stswebriskcalc). Even after matching on this composite score, we observed differences by race for the variables sex, body mass index, and smoking. We used logistic regression to test if any of these variables were associated with any of the 1-year medications listed in Table 3 and only observed that smoking was associated with insulin $(p=0.033)$, hypoglycemic $(p=0.046)$, and smoking cessation $(p=$ 0.033 ). The overall lack of association between 1-year medications and these potential confounders suggests that our results are not significantly additionally influenced by these factors.

Fisher's exact tests were performed to determine significant differences between the AI and non-AI groups while controlling for time which allows us to determine if any time points were driving racial disparities. We used the MatchIt package (logit model) for the statistical program $\mathrm{R}$ [version 3.4.2] for propensity matching to ensure that variables were similarly distributed in our sampled population between AI and Caucasian groups. Given the limited sample size rather than choosing a specific set of variables associated with medications and race, we chose the more comprehensive STS score to match on. A p-value of 0.05 was considered significant.

\section{Results}

The study population was predominantly Northern Plains Lakota, Dakota, Ojibway, and non-Hispanic whites. Preoperative clinical characteristics, patient risk factors, and operative details are detailed in Table 1. Medications at the time of discharge are listed in Table 2. The incidence of prescribed antiplatelet medication (aspirin), beta-blockers, and statin therapy was similar between AI and non-AI patients at discharge. AI patients were significantly more likely to receive insulin $(p=0.002)$ and opioids $(p=0.03)$ compared to non-AI patients at discharge, while non-AI patients received more antiarrhythmic medications $(p=0.002)$. GDMT at discharge occurred in $89 \%$ of non-AI patients and $85 \%$ of AI patients $(p=N S)$. The incidence of formal smoking cessation treatment plans was low in both groups at the time of discharge, despite higher rates of tobacco use in AIs. 
Table 1. Patient Preoperative Characteristics

\begin{tabular}{lcc}
\hline Preoperative characteristics & & \\
\hline Variable & $\begin{array}{c}\text { American Indian } \\
\%(\boldsymbol{n}=\mathbf{7 4})\end{array}$ & $\begin{array}{c}\text { Non-American } \\
\text { Indian \% }(\boldsymbol{n}=\mathbf{7 4})\end{array}$ \\
\hline Age & $60.4 \pm 11.0$ & $68.0 \pm 9.3$ \\
Sex (female) & $36.5(27)$ & $13.5(10)$ \\
Height (cm) & $173.6 \pm 9.4$ & $174.7 \pm 8.4$ \\
Weight (kg) & $93.1 \pm 19.3$ & $91.6 \pm 18.1$ \\
Body mass index & & \\
BMI category & $10.8(8)$ & $13.5(10)$ \\
$\quad$ Underweight/normal (<24.9) & $36.5(27)$ & $40.5(30)$ \\
Overweight (25.0-29.9) & $25.7(19)$ & $33.8(25)$ \\
Obese I (30.0-34.9) & $20.3(15)$ & $8.2(6)$ \\
Obese II (35.0-39.9) & $6.8(5)$ & $4.0(3)$ \\
Morbid obese (40.0+) & $63.5(47)$ & $41.9(31)$ \\
Diabetes & & \\
Diabetes control & $45.9(34)$ & $64.9(48)$ \\
None/diet & $17.6(13)$ & $27.0(20)$ \\
Oral & $36.5(27)$ & $8.1(6)$ \\
Insulin & $5.4(4)$ & $1.4(1)$ \\
Dialysis & $89.2(66)$ & $78.4(58)$ \\
Hypertension & $74.3(55)$ & $32.4(24)$ \\
Chronic lung disease & $60.8(45)$ & $20.3(15)$ \\
Smoker & $13.5(10)$ & $16.2(12)$ \\
Preop congestive heart failure & $56.8(42)$ & $41.9(31)$ \\
History of Ml & $6.8(5)$ & $14.9(11)$ \\
Arrhythmia (atrial fibrillation, & & \\
atrial flutter, other) & & \\
\hline
\end{tabular}

BMI, body mass index; MI, myocardial infarction.

Differences in secondary prevention were evident between groups 1 year after CABG (Table 3). Despite their higher risk, AI patients were less likely to be on a statin 1 year after CABG (non-AI 93\% vs. AI 81\%; $p=0.04$ ). Aspirin and beta-blocker usage 1 year after

Table 2. Thirty-Day Medications

\begin{tabular}{|c|c|c|c|}
\hline $\begin{array}{l}\text { Secondary prevention } \\
\text { measures }\end{array}$ & $\begin{array}{c}\text { Non-American } \\
\text { Indian } \\
(N=74), \\
n(\%)\end{array}$ & $\begin{array}{c}\text { American } \\
\text { Indian } \\
(N=71) \\
n(\%)\end{array}$ & $p$ \\
\hline Aspirin & $71(95.9)$ & $71(100)$ & 0.2451 \\
\hline ADP inhibitors & $14(18.9)$ & $11(15.5)$ & 0.6629 \\
\hline Statins & $69(93.2)$ & $61(85.9)$ & 0.1786 \\
\hline $\begin{array}{l}\text { Other lipid lower } \\
\text { medications }\end{array}$ & 5 (6.76) & $4(5.63)$ & 1.000 \\
\hline Beta blockers & $69(93.2)$ & $68(95.8)$ & 0.7192 \\
\hline Other antiarrhythmics & $54(73.0)$ & $30(42.3)$ & $0.0002^{*}$ \\
\hline ACEI/ARB & $41(55.4)$ & $46(63.8)$ & 0.3093 \\
\hline Diuretics & $35(47.3)$ & $30(42.3)$ & 0.6171 \\
\hline Insulin (NPH and analog) & $15(20.3)$ & $32(45.1)$ & $0.0024^{*}$ \\
\hline Oral hypoglycemics & $20(27.0)$ & $33(46.5)$ & $0.0167^{*}$ \\
\hline Other hypoglycemics & $1(1.35)$ & $2(2.82)$ & 0.6146 \\
\hline Antidepressants & 19 (25.7) & $18(25.4)$ & 1.000 \\
\hline Opioids & $64(86.5)$ & $69(97.2)$ & $0.0315^{*}$ \\
\hline Other pain medications & 29 (39.2) & $31(43.7)$ & 0.6159 \\
\hline Smoking cessation & $3(4.05)$ & $8(11.3)$ & 0.1243 \\
\hline GDMT & $66(89.2)$ & $61(85.9)$ & 0.6195 \\
\hline
\end{tabular}

${ }^{*} p<0.05$.

ACEI/ARB, angiotensin converting enzyme inhibitor/angiotensin I receptor blocker; ADP, adenosine diphosphate; GDMT, guideline-directed medical therapy; NPH, neutral protamine Hagedorn.
Table 3. One-Year Medications

\begin{tabular}{lccc}
\hline Secondary prevention & $\begin{array}{c}\text { Non-American } \\
\text { Indian }(\boldsymbol{N}=\mathbf{7 1}), \\
\boldsymbol{n}(\%)\end{array}$ & $\begin{array}{c}\text { American } \\
\text { Indian } \\
(\boldsymbol{N}=\mathbf{6 8}), \boldsymbol{n}(\%)\end{array}$ & $\boldsymbol{p}$ \\
\hline Aspirin & $63(88.7)$ & $64(94.1)$ & 0.3673 \\
ADP inhibitors & $10(14.1)$ & $12(17.6)$ & 0.6451 \\
Statins & $66(93.0)$ & $55(80.9)$ & $0.0435^{*}$ \\
Other lipid lower & $3(4.23)$ & $4(5.88)$ & 0.7146 \\
$\quad$ medications & & & \\
Beta blockers & $65(91.5)$ & $62(91.2)$ & 1.000 \\
Other antiarrhythmics & $2(2.82)$ & $1(1.47)$ & 1.000 \\
ACEI/ARB & $25(35.2)$ & $39(57.4)$ & $0.0108^{*}$ \\
Diuretics & $21(29.6)$ & $17(25.0)$ & 0.5731 \\
Insulin (NPH and analog) & $12(16.9)$ & $29(42.6)$ & $0.0014^{*}$ \\
Oral hypoglycemics & $18(25.4)$ & $25(36.8)$ & 0.1986 \\
Other hypoglycemics & $3(4.23)$ & $0(0)$ & 0.2449 \\
Antidepressants & $12(16.9)$ & $12(17.6)$ & 1.000 \\
Opioids & $13(18.3)$ & $19(27.9)$ & 0.2272 \\
Other pain medications & $25(35.2)$ & $28(41.2)$ & 0.4897 \\
Smoking cessation & $2(2.82)$ & $5(7.35)$ & 0.2679 \\
GDMT1 & $60(84.5)$ & $51(75.0)$ & 0.2054 \\
\hline
\end{tabular}

${ }^{*} p<0.05$.

CABG were similar and high in both groups. The use of dual antiplatelet therapy was low in both groups at 1 year. Beta-blocker therapy was above $90 \%$ in both groups. GDMT (defined as the combination of aspirin, statin, and beta-blocker) trended lower in AI patients than non-AI patients 1 year after CABG (non-AI $85 \%$ vs. AI $75 \%$; $p=0.20$; Fig. 1 ).

Other differences in prescribed medications existed 1 year after CABG. The use of ACE-inhibitors and ARBs was similar between the two groups at discharge (non-AI 55.4\% vs. AI 63.8\%), however, ACEI/ARB medication usage remained high in AIs while dropping significantly in non-AIs 1 year after CABG (non-AIs $35 \%$ vs. AI $57 \%$; $p<0.05$ ). Insulin use was also significantly higher among AIs. Opioid usage was numerically different between AIs and non-AIs a year after CABG (non-AI $18 \%$ vs. AI $28 \%$; $p=0.22$ ), although this trend was not statistically significant.

Non-AI subjects were more likely to be discharged to a skilled nursing facility or home with home health care compared to AI subjects, who primarily were discharged home (Fig. 2). Although not statistically significant, there appears to be a trend of fewer AIs receiving cardiac rehabilitation (CR) after CABG $(75.3 \%$ nonAIs vs. $62.0 \% \mathrm{AI}, p=\mathrm{NS}$ ).

\section{Discussion}

Guideline-derived medical therapy (GDMT) decreases morbidity and mortality in patients after CABG. ${ }^{8-10}$ Pinho-Gomes et al. define GDMT as the use of an antiplatelet agent, a statin, and a beta-blocker after CABG, 


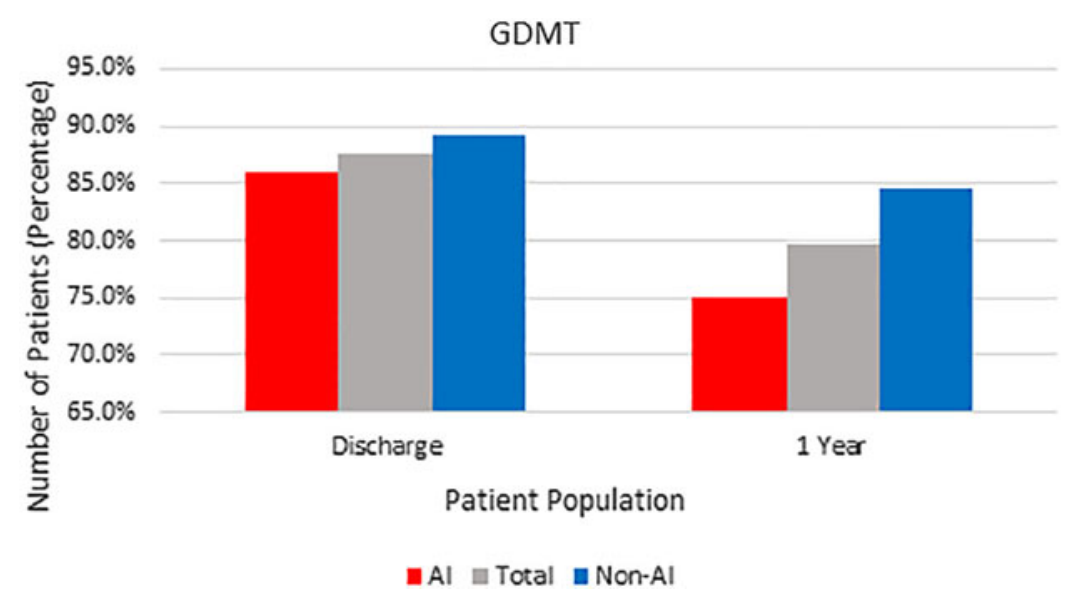

FIG. 1. Guideline directed medical therapy after coronary artery bypass grafting.

therapies known to improve graft patency, reduce cardiovascular risk, and prevent future cardiovascular events. ${ }^{9}$ However, initiation rates of GDMT after CABG vary and adherence to GDMT after CABG is challenging. For vulnerable populations with increased cardiovascular risk, GDMT becomes especially important. Compared to the general population, AIs are known to have increased incidence of cardiovascular risk factors such as smoking, diabetes, obesity, and hypertension. ${ }^{1-4}$ They are also at high risk for recurrent cardiac events after revascularization. Despite increased risk factors, CABG in AIs can be done safely, with similar short-term outcomes to the general population. ${ }^{7}$ Potential disparities in medium and long-term survival, however, make secondary prevention especially important. In this retrospective study, we have found that disparities in medical therapy exist after
CABG with the potential to adversely impact cardiovascular health in AIs.

Adherence to GDMT for secondary prevention after coronary revascularization procedures is challenging and up to a third of patients may not receive adequate prevention as soon as 1 year after surgery. ${ }^{9}$ In our study, we also found that adherence to GDMT declined in both AI and non-AIs between discharge and 1 year. While not statistically significant, adherence to GDMT trended lower in AI patients (Fig. 1) and was driven by statin usage 1 year after surgery. Determining whether this disparity in GDMT is significantly different will require further evaluation with a larger patient dataset. We did find that compliance to aspirin and betablocker therapy in both groups stayed relatively high and consistent throughout the year, perhaps because these medications are relatively inexpensive and more

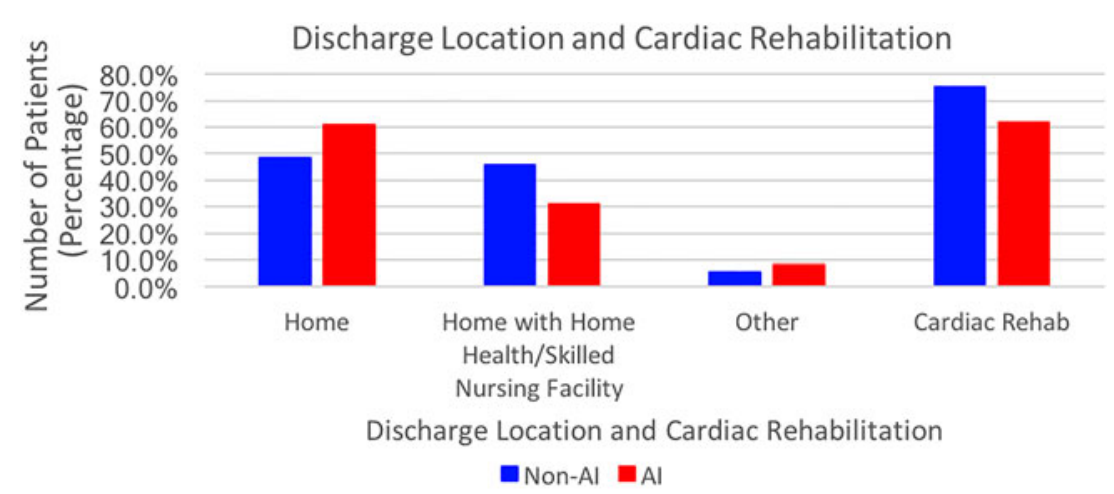

FIG. 2. Discharge location and cardiac rehabilitation after surgery. 
readily available. Statin use was lower in the AI population 1 year after CABG (non-AI 93\% vs. AI 81\% $p=0.048)$ and this trend toward lower statin use in AIs with known CAD is particularly problematic as patients with more cardiovascular risk factors would be expected to benefit most from statin therapy. These data are consistent with other studies, in which adherence to statin therapy in minority populations has been reported to be lower than the general population, although it should be noted that AIs are underrepresented in these studies. ${ }^{11,12}$ The reason for less statin usage in AIs 1 year after coronary revascularization is unclear but deserves attention as correcting this disparity would be expected to improve survival. For some patients, cost of statins may be a factor depending on the source of their prescription coverage. While generic statins are available, the difference in cost compared to aspirin and beta-blockers may be a barrier and is an area for further study. Certainly, statin therapy is cost-effective and efforts to maintain use, comparable to the general population within the AI population, are warranted.

Most AI patients receive medical care close to home in rural settings where access to health care providers can be problematic. In addition, many patients receive care through the IHSs; federal funding for IHS is lower compared to federal funding per person for other health care programs and this potentially could affect prescribing patterns. Finally, in our environment, care immediately after CABG occurs at the tertiary facility, and a year after surgery, this access to tertiary care services may be more difficult as IHS provider access and IHS access to diagnostic imaging and specialty centers is inadequate. ${ }^{13}$ We speculate that improving coordination and communication between the tertiary health care environment and local providers and healers is likely to help adherence to GDMT, improve patient survival, and improve health maintenance in this higher risk population.

In our study, AIs were more likely to have a prescription for opioids at the time of discharge and were more likely to be on opioids 1 year after surgery than nonAIs. This observation is concerning. The United States is experiencing an opioid epidemic with deaths due to opioid abuse; in 2016, it was five times higher than that in 1999, and AI mortality rates due to opioid overdose have been steadily increasing. ${ }^{14}$ In Minnesota, a state serviced by our tertiary care center, opioid overdose occurred in 47.6 deaths per 100,000 in AIs compared with 7.3 per 100,000 in non-Hispanic whites. ${ }^{15}$ Surgical specialties prescribe more opioids than most other specialties, and perioperative opioid use after surgical procedures has been shown to influence opioid use in the long-term. ${ }^{16-18}$ While postoperative pain control is important, attention to weaning from opioids and alternative pain management strategies in the weeks after CABG is imperative for all patients. In addition, we do not have data regarding preoperative opioid use in our patients, which may be relevant as the indication for opioid use a year after surgery is unclear. The reason for disparities in opioid prescribing patterns between $\mathrm{AI}$ and non-AI patients after CABG is an important topic which mandates further investigation

Hypertensive and diabetic medications were also different between AIs and the general population after CABG. AIs were more likely to be on multiple antihypertensive medications 1 year after CABG and specifically more likely to be prescribed ACE-inhibitors and ARBs. ACE-inhibitors and ARBs not only help treat hypertension but also have cardio-protective qualities that are beneficial postoperatively. ${ }^{19}$ The higher incidence of ACE-inhibitors and ARBs may reflect appropriate treatment of the increased cardiovascular risks facing AI patients. Diabetic patients are at especially high cardiovascular risk and the use of ACE-inhibitors and ARBs may help prevent diabetic nephropathy and other long-term complications of diabetes. Blood pressure control is frequently suboptimal in the preoperative and postoperative $C A B G$ patient, highlighting the need for better blood pressure control with the use of multiple medications such as beta blockers and ACEinhibitors/ARBs.

In our study, AI patients were more likely to be discharged and maintained on insulin therapy (Tables 2 and 3) after surgery. In addition, fewer oral hypoglycemics were used 1 year after CABG. Whether this reflects the severity of the disease or changes in prescribing patterns is unclear. AI patients were more likely to present on insulin therapy. ${ }^{7}$ In the immediate perioperative period, insulin therapy (frequently as intravenous infusion) is used as blood sugar fluctuations are quite common. As patients return to their local providers after surgery, an expectation would be for increasing oral hypoglycemic use as the stress of surgery diminishes. Our finding that oral hypoglycemic medication use is reduced in the AI population is consistent with earlier studies showing less effective control and reduced hypoglycemic use in AIs with diabetes. ${ }^{20}$ Whether this is driven by cost is unclear but 
a potential cause for this disparity, especially for AI patients with inadequate prescription drug coverage.

Formal CR is recommended for all CABG patients postoperatively and has been shown to improve long-term outcomes after CABG, with a $26 \%$ risk reduction in the rate of cardiovascular mortality. ${ }^{8,21}$ Furthermore, an inverse dose-response relationship exists between the number of CR sessions attended and long-term rates of myocardial infarction and death. ${ }^{22}$ Our study found that fewer AIs attended formal CR than non-Hispanic whites, although this was not statistically significant. Access to CR may be more difficult for AIs because of cost and lack of third-party payment coverage. While AI patients after CABG may value home healers and caregivers for spiritual or family reasons, combining the benefits of CR with the spiritual well-being of home care is an opportunity for improvement in the prevention of future cardiac events after $\mathrm{CABG}^{23}$ In our treatment area, there is only one tribally run nursing home facility. Increased funding to allow for more tribally run rehabilitation is likely to improve adherence to $\mathrm{CR}$ and improve the prevention of CVD.

\section{Limitations and Summary}

This was a single-center, retrospective study of a Northern Plains AI population undergoing CABG and is likely underpowered to detect differences in outcomes and treatments. The AI population of Northern Plains Indians also may not be generalizable to other AI/Alaskan Native populations as cultural, geographic, and socioeconomic differences exist between different tribal regions. However, AIs are an understudied population and little data exist after CABG. Prescribing patterns and medical care also varies regionally in the general population, as does access to tertiary care facilities.

This study highlights an opportunity to improve adherence to guideline-directed medical therapy after $\mathrm{CABG}$, reduce the recurrence of ischemic heart disease, and potentially improve long-term outcomes. While AIs may undergo CABG with excellent short-term outcomes, we identified disparities in medication and rehabilitation therapies that exist between AIs and nonAIs 1 year after surgery. Specifically, increasing statin use, improving CR, smoking cessation, and improving diabetic and hypertension management would be expected to improve long-term outcomes and are an important area for further investigation. Incorporating GDMT within the context of the local tribal environment and traditional healing is critical for acceptance.
Finally, while not related to the prevention of ischemic heart disease, differences in prescribing patterns of opioids between AIs and non-AIs are troubling and obviously impactful to AI health. Further investigation into the source of this disparity is indicated.

\section{Funding Information}

No funding was received for this article.

\section{References}

1. Howard BV, Lee ET, Cowan LD, et al. Rising tide of cardiovascular disease in American Indians: the strong heart study. Circulation. 1999;99:23892395.

2. Deen JF, Adams AK, Fretts A, et al. Cardiovascular disease in American Indian and Alaska native youth: unique risk factors and areas of scholarly need. J Am Heart Assoc. 2017;6:e007576.

3. Jernigan VBB, Duran B, Ahn D, et al. Changing patterns in health behaviors and risk factors related to cardiovascular disease among American Indians and Alaska natives. Am J Public Health. 2010;100:677-683.

4. Jernigan VBB, Wetherill M, Hearod J, et al. Cardiovascular disease risk factors and health outcomes among American Indians in Oklahoma: the THRIVE study. J Racial Ethn Health Disparities. 2017;4:1061-1068.

5. Adamsen C, Schroeder S, LeMire S, et al. Education, income, and employment and prevalence of chronic disease among American Indian/ Alaska Native elders. Prev Chronic Dis. 2018;15:E37.

6. Seligman HK, Laraia BA, Kushel MB. Food insecurity is associated with chronic disease among low-income NHANES participants. J Nutr. 2010; 140:304-310.

7. Anderson E, Glogoza M, Bettenhausen A, et al. Disparities in cardiovascular risk factors in northern plains Indians undergoing coronary artery bypass grafting. Health Equity. 2018;2:152-160.

8. Kulik A, Ruel M, Jneid $H$, et al. Secondary prevention after coronary artery bypass graft surgery: a scientific statement from the American Heart Association. Circulation. 2015;131:927-64.

9. Pinho-Gomes A-C, Azevedo L, Ahn J-M, et al. Compliance with guidelinedirected medical therapy in contemporary coronary revascularization trials. J Am Coll Cardiol. 2018;71:591-602.

10. Kulik A, Ruel M. Suboptimal medical therapy after coronary revascularization: A missed opportunity. J Am Coll Cardiol. 2018;71:603-605.

11. Lewey J, Shrank WH, Bowry AD, et al. Gender and racial disparities in adherence to statin therapy: a meta-analysis. Am Heart J. 2013;165: 665-678.

12. Johansen ME, Hefner JL, Foraker RE. Antiplatelet and statin use in US patients with coronary artery disease categorized by race/ethnicity and gender, 2003 to 2012. Am J Cardiol. 2015;115:1507-12.

13. Galloway JM. Cardiovascular health among American Indians and Alaska natives. Am J Prev Med. 2005;29:11-17.

14. Centers for Disease Control and Prevention. Opioid Overdose: understanding the epidemic. 2017. Available at https://www.cdc.gov/ drugoverdose/epidemic/index.html.

15. Tipps RT, Buzzard GT, McDougall JA. The Opioid Epidemic in Indian Country. J Law Med Ethics. 2018;46:422-436.

16. Levy B, Paulozzi L, Mack KA, et al. Trends in opioid analgesic-prescribing rates by specialty, U.S., 2007-2012. Am J Prev Med. 2015;49:409-413.

17. Zhao SZ, Chung F, Hanna DB, et al. Dose-response relationship between opioid use and adverse effects after ambulatory surgery. J Pain Symptom Manage. 2004;28:35-46.

18. Clarke H, Soneji N, Ko DT, et al. Rates and risk factors for prolonged opioid use after major surgery: population based cohort study. BMJ 2014;348.

19. Mancia G, Fagard R, Narkiewicz K, et al. 2013 ESH/ESC guidelines for the management of arterial hypertension: the task force for the management of arterial hypertension of the European Society of Hypertension (ESH) and of the European Society of Cardiology (ESC). J Hypertension 2013; 31(7):1281-1357.

20. Schmittdiel J, Steiner J, Adams A, et al. Diabetes care and outcomes for American Indians and Alaska natives in commercial integrated delivery systems: a SUrveillance, PREvention, and ManagEment of Diabetes Mellitus (SUPREME-DM) Study. BMJ Open Diabetes Res Care. 2014;2:e000043. 
21. Taylor RS, Brown A, Ebrahim S, et al. Exercise-based rehabilitation for patients with coronary heart disease: systematic review and metaanalysis of randomized controlled trials. Am J Med. 2004;116:682-692.

22. Hammill BG, Curtis LH, Schulman KA, et al. Relationship between cardiac rehabilitation and long-term risks of mortality and myocardial infarction among elderly medicare beneficiaries. Circulation. 2010;121:63-70.

23. Hodge DR, Wolosin RJ. American Indians and spiritual needs during hospitalization: developing a model of spiritual care. Gerontologist. 2014; 54:683-692.

Cite this article as: Kruger $\mathrm{H}$, Zumwalt $\mathrm{C}$, Guenther $\mathrm{R}$, Jansen $\mathrm{R}$, Warne D, Dyke C (2019) Disparities in secondary prevention of atherosclerotic heart disease after coronary artery bypass grafting in Northern Plains American Indians, Health Equity 3:1, 520-526, DOI: 10.1089/heq.2019.0030.

\section{Abbreviations Used}

$\mathrm{ACE}=$ angiotensin converting enzyme $\mathrm{ACEl}=$ angiotensin converting enzyme inhibitor $\mathrm{Al}=$ American Indian

ARBs = angiotensin II receptor blocker

$C A B G=$ coronary artery bypass grafting

$\mathrm{CAD}=$ coronary artery disease

$\mathrm{CR}=$ cardiac rehabilitation

$\mathrm{CVD}=$ cardiovascular disease

GDMT = guideline-directed medical therapy

$\mathrm{IHS}=$ Indian Health Service

$\mathrm{NPH}=$ neutral protamine Hagedorn

NS $=$ not significant

STS $=$ Society of Thoracic Surgeons

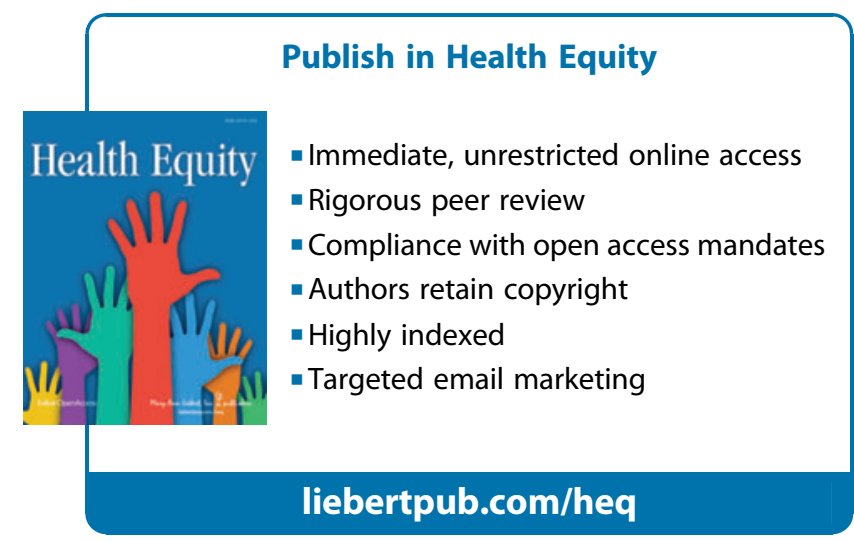

